\section{Ultrasensitive MAPK/Erk activation in absence of protein synthesis in Xenopus oocytes}

\author{
Rémy Beaujois,1,2 Franck Riquet,1,2 \\ Katia Cailliau, ${ }^{1}$ Edith Browaeys-Poly, ${ }^{1}$ \\ Christophe Russo, 1,2 Ralf Blossey, 2 \\ Dorothée Vicogne,2 Matthieu Marin, ${ }^{1}$ \\ Arlette Lescuyer-Rousseau, 1 \\ Jean-Pierre Vilain, 1 \\ Jean-François Bodart1,2
}

1EA 4479, Laboratoire de Régulation des Signaux de Division, University of Lille1; 2USR 3078 CNRS, Institut de Recherche Interdisciplinaire, Villeneuve d'Ascq, France

\begin{abstract}
The mitogen-activated protein kinase (MAPK) cascade in Xenopus oocytes exhibits an all-or-none, ultrasensitive response, which is believed to result from a positive feed-back loop. Here we describe a context where 1,10-phenanthroline slowly, but strongly, activates MAPK while it impairs protein synthesis in a zincdependent manner, abolishing any feed-back loop. The induced-MAPK response was found to be strongly ultrasensitive. We argue that underlying this behaviour is a regulation motif akin to a feed-forward loop acting in vivo.
\end{abstract}

\section{Introduction}

A specific All-or-None response for mitogenactivated protein kinase/extracellular signalregulated kinase (MAPK/Erk2/Xp42Mpk1) activation is observed in Xenopus oocytes at the cell cycle G2/M transition. G2-synchronized oocytes enter M-phase upon stimulation of Ras-independent [progesterone (PG)] or Rasdependent [insulin, heterologously expressed fibroblast growth factor-receptor (FGF-R)] pathways. The MAPK response, in this context, contrasts with the gradual answer of the MAPK cascade to external stimuli in somatic cells. The oncoprotein Moloney sarcoma (Mos) is an oocyte-expressed kinase, which functioned ancestrally in regulating specialization of female meiosis. ${ }^{1}$ Mos plays a pivotal role as the upstream activator of the mitogen activated protein kinase kinase (MAP2K)-extracellular regulated kinase (MAPK/Erk) cascade in Xenopus oocytes. ${ }^{2-3}$ Once accumulated, Mos activates MAP2K, which in turn phosphorylates MAPK. One has to note that Erk2/Xp42Mpk1 is the only Erk expressed in Xenopus oocytes.
Observed physical properties of MAPK activation in Xenopus oocytes are ultrasensitivity, bistability and irreversibility.4-5 Ultrasensitivity has been defined to indicate cases in which the sensitivity of a system to a signal is greater than that expected from standard hyperbolic (Michaelis-Menten) response. Sensitivity can be expressed quantitatively in terms of a Hillcoefficient. ${ }^{6}$ The Erk signalling cascade organisation generates the steepness of Erk response in Xenopus oocytes. Both bistability and ultrasensitivity are related to the existence of positive feed-back loop motifs within the network promoting Mos synthesis $7-8$ or enhancing Mos stability by phosphorylation, ${ }^{9}$ which might be achieved by MAPK ${ }^{10}$ or by M-phase promoting factor/cyclin dependent kinase (Cdk1)-cyclin B complex (MPF). ${ }^{11}$ Other regulation motifs, such as a feed-forward loop, ${ }^{12}$ may be involved in the building of an ultrasensitive response.

A feed-forward loop (FFL) is a three-nodes motif that appears in many transcription regulatory networks, where A activates $\mathrm{B}$ while A and $B$ activate $C$. According to their types, FFLs act to filter noisy input signals, accelerate signal by speeding up the target response, build transitory responses like pulses or even delay signals. ${ }^{13-14}$ For instance, in budding yeasts, FFLs are intimately linked in the initiation of DNA synthesis and cell division at the G1/S and M/G1 transitions. ${ }^{12,15}$ Also, in eukaryotic cells, FFLs are believed to play a crucial role in converting single oscillatory signals into coordinated events, and are involved in driving the activation of Cdk-cyclin pairs into a robust temporal sequence. 12

Here we tested the hypothesis that an ultrasensitive response may be generated for MAPK response in absence of feed-back loop, through other regulation motifs, and seek for experimental conditions where MAPK could be activated in absence of feed-back. To this purpose, zinc-chelator 1,10-Phenanthroline monohydrate (1,10-PA) was used, based on earlier observations reporting this chelator of zinc to be a potential activator of MAPK in Xenopus 0ocytes. 16 The present work reports characterization of the molecular mechanisms leading to an ultrasensitive activation of MAPK in absence of the classical feed-back loops promoted by MPF activation or Mos accumulation.

\section{Material and Methods}

\section{Handling of oocytes}

Adult Xenopus females were purchased at University of Rennes I (France). After anesthetizing Xenopus females by immersion in 1 g.L.-1 MS222 solution (tricaine methane sulfonate), ovarian lobes were surgically removed and kept physiological in ND96 medium (96
Correspondence: Jean-François Bodart, EA 4479, Laboratoire de Régulation des Signaux de Division, Building SN3, University Lille1, F59655 Villeneuve d'Ascq, France.

Tel.: +33.3.2043.6867 - Fax: +33.3.2043.4038. E-mail: jean-francois.bodart@univ-lille1.fr

Key words: MAPK, ultrasensitivity, feed-back, protein synthesis, regulation motif.

Acknowledgements: R. Beaujois was MRT grant recipient. C. Russo was recipient of a grant from INCa (Institut National contre le Cancer).

Conflict of interests: the authors declare that they have no conflict of interests.

Received for publication: 17 September 2012. Revision received: 17 September 2012.

Accepted for publication: 17 September 2012.

This work is licensed under a Creative Commons Attribution NonCommercial 3.0 License (CC BYNC 3.0).

(C) Copyright R. Beaujois et al., 2013

Licensee PAGEPress, Italy

MAP Kinase 2013; 2:e2

doi:10.4081/mk.2013.e2

$\mathrm{mM} \mathrm{NaCl}, 2 \mathrm{mM} \mathrm{KCl}, 1.8 \mathrm{mM} \mathrm{CaCl} 2,1 \mathrm{mM}$ $\mathrm{MgCl}_{2}, 5 \mathrm{mM}$ HEPES-NaOH, pH7.5). Fullygrown stage $\mathrm{VI}$ oocytes were isolated and follicle cells were removed by a collagenase treatment for $30 \mathrm{~min}$ ( $1 \mathrm{mg} \mathrm{mL}^{-1}$ collagenase A) followed by a manual microdissection using forceps. Oocytes were stored at $14^{\circ} \mathrm{C}$ in ND96 medium until use.

\section{Xenopus oocyte treatment}

Oocytes were obtained as stated in Supplementary Information. They were incubated in ND96 medium containing $35 \mu \mathrm{M}$ cycloheximide (CHX) (in ethanol-0H), $50 \mu \mathrm{M}$ U0126 (in DMS0). These overnight incubations were performed before mRNA injection and/or incubation in PG (12 $\mu \mathrm{M})$ and/or 1,10PA $(1 \mathrm{mM})$ and/or $\mathrm{ZnSO}_{4}(0.3 \mathrm{mM})$. Using syringe positive displacement digital micropipette (Nichiryo, Tokyo, Japan), mRNA microinjections were performed in the equatorial region (50-55 ng of GFP-CL4 mRNA $1 \mu \mathrm{g} / \mathrm{\mu L}$ or $60 \mathrm{ng}$ of FGF-R mRNA $1 \mu \mathrm{g} / \mu \mathrm{L}$ per oocyte). Control oocytes were incubated or injected with an identical amount of DMSO (1\%0) or ethanol (1\%0) or water (50 to $60 \mathrm{~nL} / 00$ cyte).

\section{Stimulation and analysis of $\mathrm{G} 2 / \mathrm{M}$ transition (meiotic resumption)}

M-phase entry was induced by incubating G2-arrested oocytes in ND96 medium containing $12 \mu \mathrm{M}$ of progesterone. All experiments were performed at $19^{\circ} \mathrm{C}$. Kinetic of germinal 
vesicle breakdown (GVBD) was scored by the appearance of a white spot (WS) at the animal pole of the cell attesting of the M-phase entry. For each point of kinetics, batches of ten oocytes representative for GVBD percentage were frozen at $-20^{\circ} \mathrm{C}$ for later biochemical analysis.

\section{Chemicals and bioreagents}

Collagenase A and protease inhibitors were purchased from Roche Applied Science (Meylan, France). Mouse monoclonal anti-Erk2 (D-2) and rabbit polyclonal anti-Mos ${ }^{\mathrm{xe}}$ (C-237), anti-Raf (C-20) and anti-Actin (I-19) antibodies were obtained from Santa Cruz Biotechnologies (Heidelberg, Germany). Mouse monoclonal anti-GFP was purchased from Roche Applied Science, mouse monoclonal anti-PDGF-R from BD Transduction Laboratories ${ }^{\mathrm{TM}}$ (Le Pont de Claix, France), rabbit polyclonal anti-phosphoTyr15-Cdc2 antibody from Cell Signaling Technology ${ }^{\mathrm{TM}}$ (Saint Quentin en Yvelines, France) and rabbit polyclonal anti-active jun $\mathrm{N}$ terminal protein kinase (JNK) pAb and antiphosphoMEK1/2 (pSer218/222) antibodies were purchased from Promega (Charbonnière-lesBains, France) and Merck-Calbiochem (Nottingham, England), respectively. Rabbit polyclonal anti-CyclinB2 (JG103) is a gift from Dr. J. Gannon (ICRF, South Mimms, UK). Tricaine methane sulfonate, progesterone (Pg), 1,10-phenanthroline monohydrate (1,10-PA), zinc sulphate $\left(\mathrm{ZnSO}_{4}\right)$, protein synthesis inhibitor CHX, and MEK inhibitor U0126 ethanolate and Bovine serum albumin were obtained from Sigma (Milwaukee, WI, USA). MAPKinase (ERK1/2) activity assay kit was purchased from Millipore ${ }^{\mathrm{TM}}$ (Billerica, MA, USA). ECL and hyperfilms from GE Healthcare Life Sciences (Orsay, France) were used for western blotting.

\section{Plasmid constructs and in vitro transcriptions}

Regarding GFP-Clone4 probe (GFP-CL4), construction of the pCS2-memb-eGFP plasmid as initially described by Larson, 17 was achieved using the pCS2-memb-mCherryl8 by swapping fluorescent proteins coding sequences, mCherry for eGFP. The eGFP coding sequence fragment was derived from the pEGFP-1 (Clontech, Saint-Germain-en-Laye, France). Plasmid were propagated in XL1 blue (Stratagene, Massy, France) and checked by sequencing. mRNAs encoding the memb-EGFP were transcribed in vitro using mMessage mMachine Sp6 kit (Ambion, Courtaboeuf, France) according to manufacturer's recommendations. Briefly, $1 \mu \mathrm{g}$ of Not I digested pCS2-memb-eGFP template was incubated in the reaction mixture at $37^{\circ} \mathrm{C}$ for $2 \mathrm{~h}$ followed by a 15' treatment with DNAse I. Transcripts were subsequently cleaned using spin column
(Chroma Spin-100, Clontech). Quality of RNA preparations were assessed on agarose gel and concentrations were determined by spectrophotometry using a NanoDrop (ND-1000, Agilent Technologies, Massy, France). As described by Cailliau et al., ${ }^{19}$ FGF-R probe is a chimera composed of the extracellular domain of the human PDGF-R and the intracellular domain of the $P$. waltlii FGF-R1 (gift from Dr. D.L. Shi). Linearised from full-length cDNAs inserted into vector pSP64T,20-21 capped mRNAs encoding the chimera were transcribed as specified for GFP-CL4.

\section{Oocytes lysis and preparation of protein samples}

Oocytes were lysed at $4^{\circ} \mathrm{C}$ in homogenization buffer ( $60 \mathrm{mM} \mathrm{2-} \mathrm{glycerophosphate,} 15$ $\mathrm{mM}$ paranitrophenylphosphate, $25 \mathrm{mM}$ MOPS, $15 \mathrm{mM}$ EDTA, $15 \mathrm{mM} \mathrm{MgCl}, 2 \mathrm{mM}$ DTT, $1 \mathrm{mM}$ $\mathrm{Na}_{3} \mathrm{VO}_{4}, 1 \mathrm{mM} \mathrm{NaF}, 1 \mathrm{mM}$ phenylphosphate, and proteases inhibitors, $\mathrm{pH} 7.2)^{22}$ and centrifuged at $10,000 \mathrm{~g}$ for $15 \mathrm{~min}\left(4^{\circ} \mathrm{C}\right)$ to eliminate yolk and membranous pellet.23 For detection of chimera receptor probe (PDGFRFGFR1), 0ocytes were homogenised, centrifuged and the membrane fraction was solubilised in buffer added with Triton X100 1\%, at $4^{\circ} \mathrm{C}$. Before heating $\left(100^{\circ} \mathrm{C}, 5 \mathrm{~min}\right)$, lysates were centrifugated at $10,000 \mathrm{~g}$ for $15 \mathrm{~min}$ $\left(4^{\circ} \mathrm{C}\right)$ and liquid fraction were finally mixed with one volume of Leammli sample buffer containing 4\% -mercaptoethanol (Biorad, Marnes-la-Coquette, France).

\section{SDS-PAGE and western blotting}

Proteins were run on a 12,5\% SDS-PAGE for Mos, P-Tyr15-Cdc2, GFP probe, Actin, PMEK1/2(pSer218/222) and P-JNK1/2 detections, on a 7.5\% SDS-PAGE for PDGFR-FGFR1 probe detection, or on a $15 \%$ modified SDSPAGE for MAPK/Erk2, CyclinB2 and Raf. As described by Chesnel et al., ${ }^{24}$ modified polymerisation allows a better discrimination between the active and the inactive forms of these proteins. The equivalent of a third of an oocyte was loaded per lane and electroblotted onto nitrocellulose sheet. While the quantity of proteins remains rather constant in Xenopus oocytes, transfer efficiency and equal loading were checked using Ponceau red staining and Actin western blots. Membranes were saturated with $5 \%(\mathrm{w} / \mathrm{v})$ non-fat milk or $10 \%$ bovine serum albumin (w/v) in TBS (Tris-buffered saline)-Tween (50 mM Tris, $150 \mathrm{mM} \mathrm{NaCl}$, $0.1 \%(\mathrm{v} / \mathrm{v})$ Tween20, pH7.4) for $45 \mathrm{~min}$. Rabbit polyclonal anti-Mosxe (1:500), anti-phosphoTyr15-Cdc2 (1:5000), anti-MEK (1:500), antiRaf (1:500), anti-Active JNK pAb (1:5000), anti-Actin (1:500) and Mouse monoclonal antiPDGF-R (1/5000) primary antibodies were incubated over-night at $4{ }^{\circ} \mathrm{C}$. Mouse monoclonal anti-Erk2 (1:500) and anti-GFP (1:500), rab- bit polyclonal and anti-Cyclin B2 (1:1250) antibodies were incubated $2 \mathrm{~h}$ at room temperature. Then, membranes were washed three times for 10 min in TBS-Tween and incubated $1 \mathrm{~h}$ with either an anti-mouse (IgG or $\operatorname{IgM}$ ) horseradish peroxidase-labeled secondary antibody or an anti-rabbit IgG at a dilution of 1:2500. Finally, three washes of $10 \mathrm{~min}$ each were performed with TBS-Tween and the detection was carried out with enhanced chemiluminescence on hyperfilms. Classical ECL was used to enhance luminescence, excepted for PDGF-R antibodies (Advanced ECL Detection system, Amersham).

\section{Histological analysis}

Oocytes were fixed overnight in Smith's fixative (10\% formalin, $2.5 \%$ acetic acid, 0,5\% $\mathrm{K}_{2} \mathrm{Cr}_{2} \mathrm{O}_{7}$ ), dehydrated, and embedded in paraffin for cytological studies. $7 \mu \mathrm{m}$ sections were stained with nuclear red for detection of nuclei and chromosomes, whereas picroindigocarmine was used to reveal cytoplasmic and microtubular structures. This method is precise enough to detect spindles and condensed chromosomes, even if not located near the plasma membrane.22

\section{Quantification procedures and data processing}

MAPK activity was assessed following manufacturer instructions of STAR ERK1/2 Elisa Kit (Millipore). Films from (i) western blotting on population of oocytes (GFP-CL4 and FGF-R accumulation) and (ii) Oocyte by oocyte MAPK phosphorylation state analysis were scanned. Semi-quantification was done by Optical Density (O.D.) measurement using Quantity One V4.21 1-D gel analysis software from BioRad.

Using fluorescent probe, analysis was set thanks to Nikon Eclipse 80i microscope and objective APO 4x. Individual oocytes of each condition were pictured with Photometrics ${ }^{\circledR}$ CoolSNAPTM ES2 camera and NIS Element V2.3 imaging software. Pictures were submitted to ImageJ software analysis.

To quantify GFP, PDGFR-FGFR1, MAPK and phospho-MAPK in Western Blot, similar rectangular areas were first made to surround each band of blots. Then Volume Intensity was quantified for every plot, corresponding to the intensity data (probe 0.D.) inside a defined boundary drawn on our image. Local Background was distinguished and defined as the intensity of the non-data background pixels included in your volume quantification after area drawing. Data treatment was multi-step: (i) Local Background was subtracted from Volume Intensity for each plot, (ii) global gel background was subtracted from every volume intensities (usually 0.D. from untreated oocytes sample), (iii) normalization was done (GFP or FGF-R O.D.=1) rela- 
tively to standardized positive control sample (i.e. GFP-CL4 or FGF-R mRNA injected control oocytes). Concerning MAPK/Erk phosphorylation state, the percentage of phospho-MAPK was determined in every cell. For each plot, we only subtracted local background from volume intensity before to apply: \% p-MAPK=pMAPK/(MAPK+p-MAPK).

Fluorescence quantification was standardized and strictly performed on animal cortex where pigmentation highly diminishes cell auto-fluorescence. For each measure, dish background was subtracted to the oocyte fluorescence intensity, and background depleted values were averaged into conditions. We achieved data normalization relatively to positive control sample (GFP-CL4 Fluo=1).

\section{Statistical analysis and Hill coeffi- cient determination}

Representative western blot analysis is provided for each condition. Experiments were performed at least in triplicate. Fluorescence values are given as mean \pm standard error of the mean (S.E.M) with $\mathrm{N}=9$, where $\mathrm{N}$ is the number of oocytes and $B=4$, where $B$ is the number of batches. Statistic significance was assessed using One Way ANOVA, Tukey post hoc and accepted as ${ }^{*}(\mathrm{P}<0.05),{ }^{* *}(\mathrm{P}<0.01)$ and ${ }^{* * *}(\mathrm{P}<0.001)$.

The Hill coefficient discriminates cell response in function of variable stimulation. ${ }^{25}$ The experimental curve has been fitted using the following function: $F(x)=\left[\left(V \cdot x^{n}\right) /\left(x^{n}+\right.\right.$ $\left.\left.a^{n}\right)\right]+b$. In this function, we chose $V$ and $b$ to symbolise maximal $(\mathrm{V}+\mathrm{b})$ and minimal (b) values obtained in phospho-MAPK. $\mathrm{F}(\mathrm{x})$ corresponded to the MAPK occurrence in percent of phospho-MAPK; $x$, to the concentration in 1,10 PA (mM); a, to the Ka; $\mathrm{n}_{\mathrm{H}}$, to the Hill coefficient. Theoretical estimation of the Hill coefficient is determined by the mean of Gnuplot software. The latter own a fitting command based on the nonlinear least-squares Marquardt-Levenberg algorithm to infer the parameters values. The process consists at three mains steps: i) describe a user-defined analytical function with undetermined parameters; ii) provide this function to the fit command and iii) choose the variable parameters of interest. The output of this command gives information about the value of the desired parameter, its standard deviation error and a confidence interval.

\section{Results and Discussion}

MAPK activation induced by $1,10-P A$

Increasing concentrations of $1,10 \mathrm{PA}$ were applied to immature oocytes (Figure 1). Fortyeight hours post-incubation, 1 mM 1,10-PA acti- vated $50 \%$ of MAPK and $2 \mathrm{mM}$ drives the phosphorylation of majority of MAPK (Figure 1A). MAPK activity was assessed and kinase maximum activity was observed $72 \mathrm{~h}$ post $1,10 \mathrm{PA}$ addition (Figure 1B). Surprisingly, while the entire pool of endogenous MAPK was not recruited, activity of the kinase was even higher $(3.22 \pm 0.34$ fold) than in PG-treated control oocytes in which MAPK were fully activated (Figure 1B).

\section{1,10 PA acts through MAP2K but not MAP3K}

To decipher the mechanism underlying the MAPK strong activation in 1,10-PA action, several strategies were used including chelating competition $\left(\mathrm{ZnSO}_{4}\right)$ and MEK inhibition (U0126) prior to PG or 1,10-PA addition (Figure 1C). Because Mos accumulates, MAPK is phosphorylated and Cdk1, MPF catalytic subunit, is
A

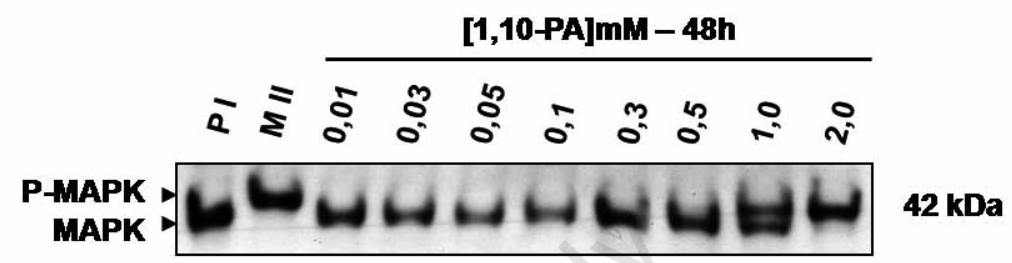

B

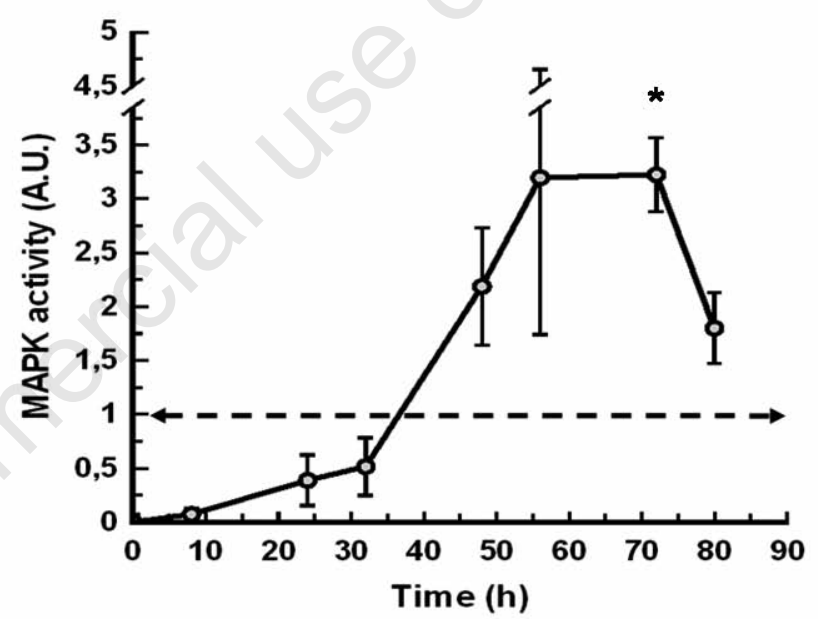

C

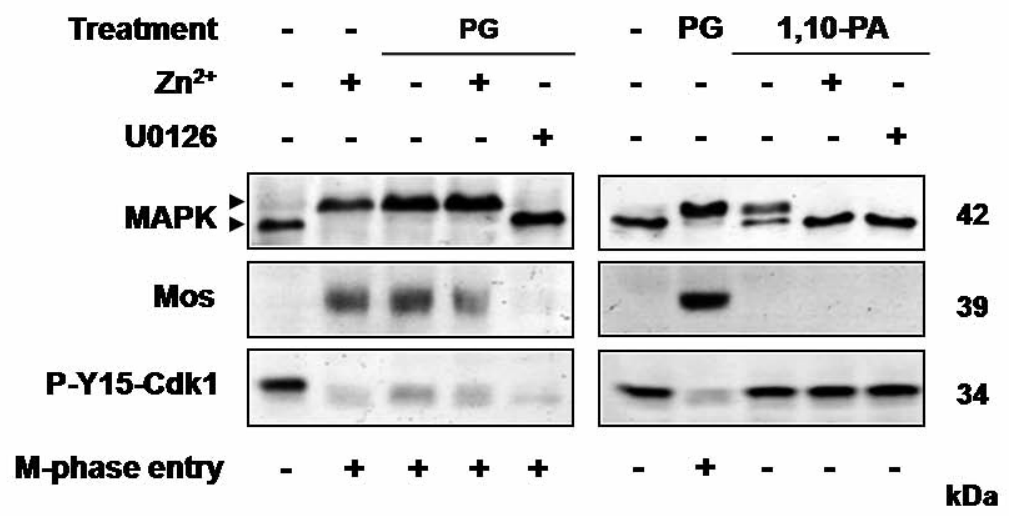

Figure 1. MAPK activation induced by 1,10-PA. 1,10-PA increasing concentrations were applied and MAPK/Erk phosphorylation profile was examined (A) and measured for 1 mM (B). Values were compared to control oocytes (PG+24 h, dotted line). Statistically significant difference was observed between MAPK activity $32 \mathrm{~h}$ vs $72 \mathrm{~h}\left(\mathrm{P}=0.044^{*} 32 \mathrm{~h}\right.$ vs $72 \mathrm{~h})$. (C) MAPK, Mos, Cdk1 were analyzed following treatment $\mathrm{Zn}^{2+}\left(\mathrm{ZnSO}_{4}\right.$, competitor) or U0126 (MAP2K) in cells either stimulated by PG or 1,10-PA. Patterns were correlated to $M-P h a s e$ entry. 
dephosphorylated on Tyr15, both MAPK and MPF pathways were activated upon progesterone stimulation (Figure 1C). M-phase entry, as attested by nuclear envelope dissolution (GVBD), was reached within 16-18 h. In 1,10-PA treated oocytes, Mos did not accumulate and MPF stayed inactive since Tyr 15 remained phosphorylated. Absence of MPF activation in this context was presumably due to $\mathrm{Cdc} 25$ inhibition. Indeed, Cdc25, which contributes to MPF activation through Tyr 15 dephosphorylation, has been shown to bind to $\mathrm{Zn}^{2+} .26-27$ The latter was required as a structural regulator for Cdc25C substrate recognition and to be critical for MPF activation at the onset of M-phase. ${ }^{27}$

Since Mos did not accumulate, we further tested the hypothesis that MAPK activation in 1,10-PA could rely on another upstream effector such as MAPK kinase kinase (MAP3K) Raf, but the latter was never observed to be phosphorylated in 1,10 PA treated oocytes (Figure 2). ${ }^{2} \mathrm{PG}$ induced GVBD and MPF activations were not impaired by MAP2K inhibitor U0126 whereas phosphorylation of MAPK was abolished as expected. Both $\mathrm{ZnSO}_{4}$ and U0126 prevented 1,10-PA-induced MAPK activation (Figure 1C), leading to the conclusion that activation of MAPK induced by 1,10 PA depends upon MAP2K but not MAP3K. We also observed that 1,10 PA induced typical morphological changes without clear dissolution of nuclear envelope. This phenotype was likely related to the effects of zincchelation rather than to MAPK activation since U0126 did not prevent the occurrence of these phenotypes (Figure 3).

\section{mRNA expression is impaired by $1,10 \mathrm{PA}$}

Absence of Mos accumulation in presence of MAPK activity is surprising, since the cascade is embedded in a positive feedback loop. Such absence of accumulation might be related to inhibitory effects of zinc chelation on protein synthesis. Therefore, the effects of 1,10-PA on protein synthesis were analysed (Figure 4). To this purpose, membrane-tethered GFP (GFPCLA) and FGF-R polyadenylated mRNA were used. Expressed proteins were immunoblotted and semi-quantified. Prophase arrested oocytes were injected with $\mathrm{H}_{2} \mathrm{O}$, GFP-CL 4 or FGF-R mRNA. Injected oocytes were either untreated or incubated in PG, 1,10-PA, or both. In absence of PG stimulation, both GFP-CL4 and FGF-R were synthesised following mRNA injection attesting for translation efficiency of polyadenylation of heterologous mRNA. As expected, CHX inhibited both GFP and FGF-R accumulation. Strong expression was observed in condition GFP-CL4 and PG+GFP-CL4, but not in other conditions where dramatically diminished levels were observed. Using positive control conditions for standardization, fluorescence signals were quantified (Figure 4B, black bars) and reported with western blot semi-quantifications

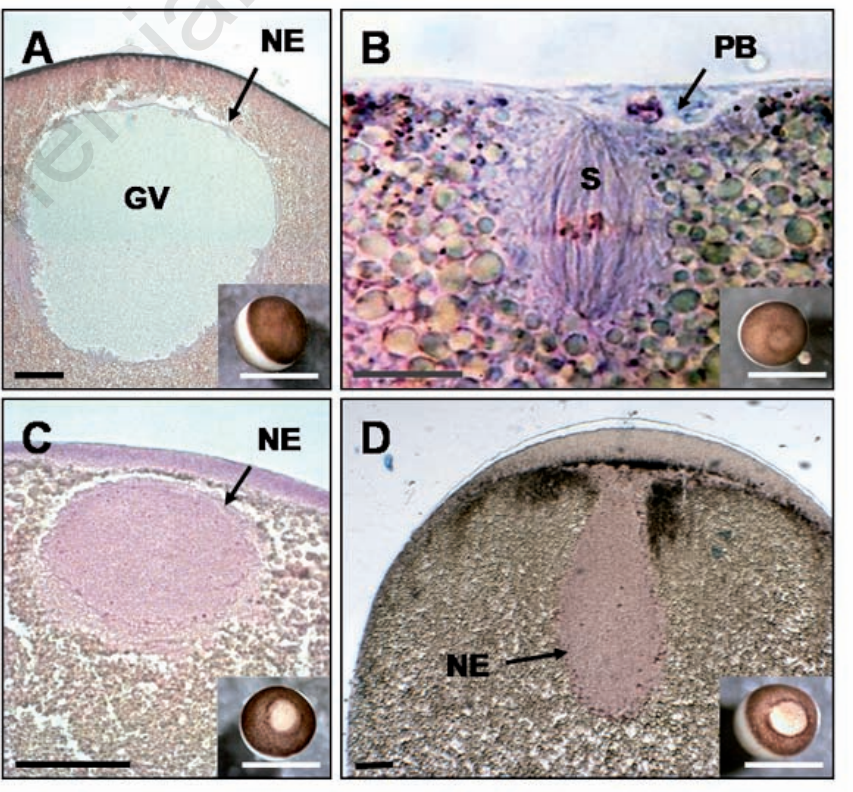

Figure 3. Morphological changes induced by 1,10 PA. Histological sections were performed. Oocyte maturation is characterized by the dissolution of nuclear envelope (germinal vesicle breakdown/GVBD), condensation of chromosomes and formation of bipolar spindle at the plasma membrane. Prophase I oocytes exhibited intact germinal vesicle while metaphase II spindle were observed in PG-treated oocytes (A vs B). 1,10-PA treated oocytes displayed atypical depigmentation at the animal cortex, which might be assimilated to white spot $(\mathrm{C})$, in a cell phenotype we here referred as a Phenan-Type. In these oocytes, GV migration was observed toward the membrane plasma, though nuclear envelope fragments were still detected and no clear GVBD was observed. Such phenotype was not altered by U0126 (D) leading us to conclude its occurrence was not dependent upon MAPK phosphorylation and activation but rather related to effects of zinc chelation on cytoskeleton. It is to note that morphological events induced by 1,10 PA were correlated to $\mathrm{p} 49 \mathrm{JNK} 2$, which was totally cancelled by $\mathrm{ZnSO} 4$ addition. NE, nuclear envelop; GV, germinal vesicle; $P B$, polar body, $S$, spindle. 
for GFP (grey bars) and FGF-R (white bars). Compared to negative control conditions, mRNAs-injected cells treated or not by PG exhibited strong accumulation of probes while CHX drastically inhibited the synthesis of proteins GFP-CL $A$ and FGF-R. 1,10-PA treated cells exhibited a low rate of protein synthesis (GFPCL4 (Fluo) $=15.64 \%$ and $20.65 \%$ respectively). No significant difference was observed between oocytes injected with GFP-CLA mRNA treated by $\mathrm{PG}$ or not $[\mathrm{P}=0.571$, GFP-CLA (Fluo)]. Indeed, when GFP-CL4-injected oocytes were treated by 1,10 PA, synthesis levels were dramatically reduced in comparison to untreated ones $\left(\mathrm{P}<0.001^{* * *}\right)$. 1,10-treated oocytes exhibited levels of fluorescence not statistically different from those of negative control $(\mathrm{P}=0.571)$. Protein synthesis was decreased by at least $80 \%$ in presence of 1,10-PA, in a dose-dependent manner (Figure 5). Increasing $\mathrm{ZnSO}_{4}$ concentrations were applied to 1,10 PA treated oocytes, to overcome the effects of zinc chelation (Figure 4C, D). $\mathrm{ZnSO}_{4}$ were not used alone since it can induce G2/M transition in absence of hormonal stimulation. ${ }^{28}$ Minimizing a role of $\mathrm{Zn}^{2+}$ per se, oocytes exposure to other divalent cations leads to spontaneous M Phase entry: Mphase entry in Rana temporaria and Xenopus laevis oocytes is induced by solutions containing $\mathrm{Mn}^{2+}$ or $\mathrm{Co}^{2+}$ ions, ${ }^{29}$ or may occur spontaneously when external $\mathrm{Ca}^{2+}$ orMg2+ ions concentrations are raised in presence of ionophore ${ }^{30}$ Nevertheless, in our conditions, $0.2 \mathrm{mM}$ and $0.3 \mathrm{mM} \mathrm{ZnSO}_{4}$ reverted 1,10-PA effects on MAPK phosphorylation and protein synthesis inhibition. In such conditions, GFPCL4 accumulated but not Mos, as expected in absence of PG stimulation. These observations correlated with immunoblot and fluorescence quantifications (Figure 4D). $\mathrm{ZnSO}_{4} 0.2 \mathrm{mM}$ and $0.3 \mathrm{mM}$ restored GFP-CLA synthesis and completely inhibited the morphological features induced by $1,10 \mathrm{PA}$ occurrence while $50 \%$ of these morphological features were vanished by $\mathrm{ZnSO}_{4} \quad 0.1 \mathrm{mM}$. Statistical differences were noted between values from conditions $\mathrm{ZnSO}_{4}$ $0.2 \mathrm{mM}$ and $0.3 \mathrm{mM}$ versus $\mathrm{Zn}^{2+} 0 \mathrm{mM}$ and 0.1 $\mathrm{mM}$ (not shown). Thus, 1,10-PA inhibitory effects on protein synthesis relies on zinc chelation: zinc chelation by $1,10-\mathrm{PA}$ is known to prevent cytoplasmic polyadenylation element binding protein (CPEB), a crucial enzyme in polyadenylation processes, from binding to RNA and therefore to promote protein synthesis. ${ }^{31}$ CPEB inhibition may explain the absence of Mos accumulation, since its mRNA has to be unmasked by polyadenylation in order to be translated. Nevertheless, the mechanisms involving zinc and impairing the synthesis of our probes remain to be elucidated, but may involve inhibition of eIF-factors, which require zinc to initiate translation. ${ }^{32-33}$ One might not discard that 1,10 PA may impact protein synthesis through other metal-ion sequestration.

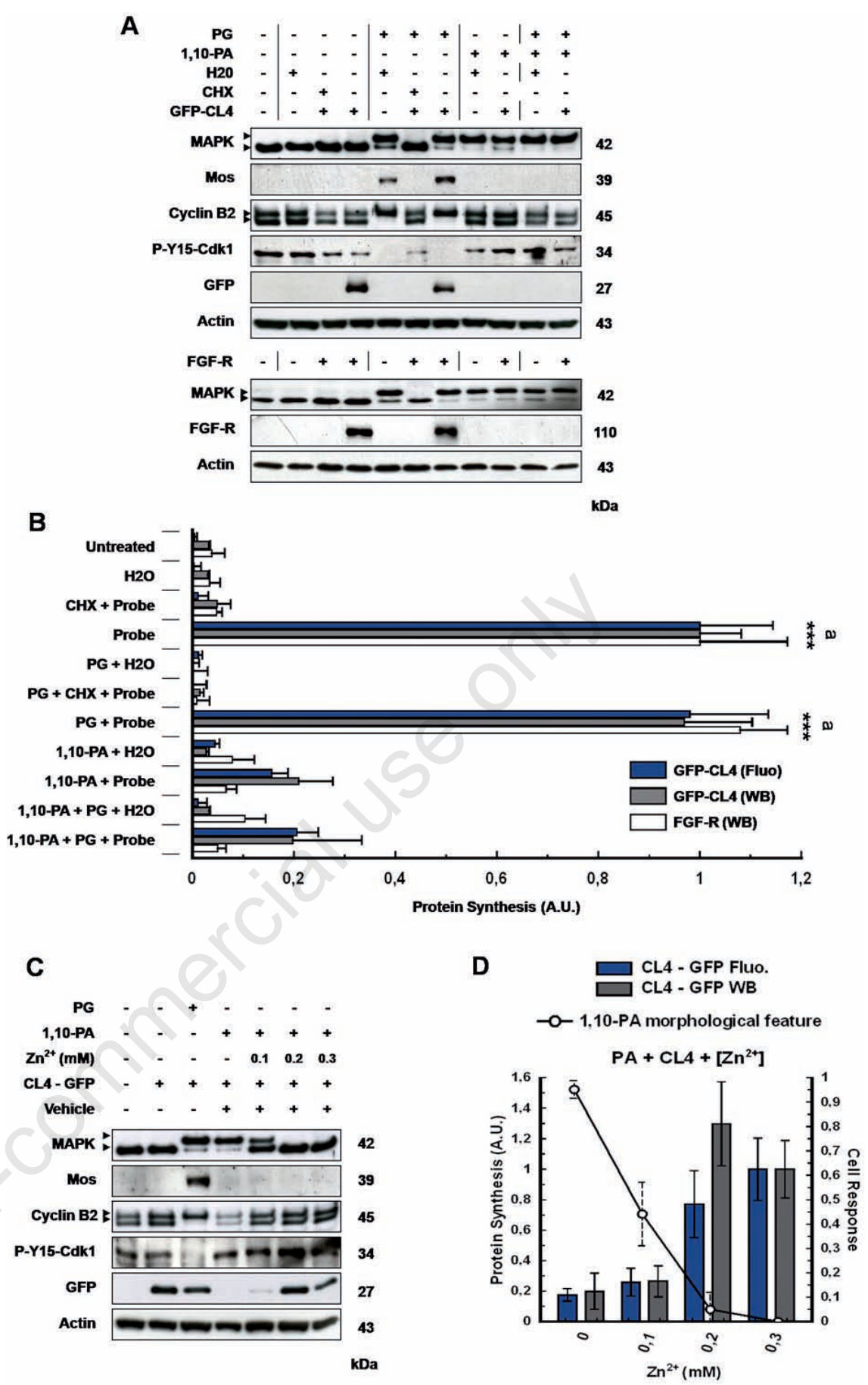

Figure 4. 1,10-PA dramatically inhibits protein synthesis in spite of MAPK strong activity. Protein synthesis was measured by western blotting and fluorescence microscopy by fluorescence detection at animal cortex where pigmentation overcomes platelets auto-fluorescence (A) following H20 or polyadenylated mRNA (GFP-CL4 and FGF-R) injections, and upon PG or 1,10-PA treatment. $\mathrm{CHX}$ was used as a negative control. Cell lysates were subjected to SDS-PAGE for MAPK, CyclinB2, Cdk1 phosphorylation states and Mos, GFP-CL4 and FGF-R accumulation profiles. (B) Plasma membrane fluorescence quantification (black bars), GFP-CL4 (grey bars) and FGF-R (white bars) semiquantifications from immunoblot were reported and submitted to statistical assay. Increases in $\mathrm{ZnSO}_{4}$ were applied to 1,10-PA treated oocytes (C-D) GFP-CL4 probe accumulation was evaluated quantified by microscopy (blue bars) and immunoblot (grey bars) correlatived to cell responses: M-phase entry or typical morphological features induced by zinc chelation (pigment rearrangements). Arbitrary Units (A.U.) were used to report protein synthesis. 


\section{MAPK ultrasensitive response without feedback loop}

Based on our observations, it can be assumed that MAPK activity measured upon 1,10-PA addition arose in absence of any feed-back loop since protein synthesis and MPF activity were inhibited in this experimental context. Regarding the MAPK profile of partial activation in 1,10 PA-treated oocytes, two hypotheses may be considered: i) both states of MAPK (phosphoMAPK and unphospho-MAPK) are found together in individual oocytes, according to a gradual response type or ii) isoforms are found in separate oocytes, according to the all-or-none response paradigm. Therefore, MAPK phosphorylation profiles were then determined cell by cell, i.e. on $\mathrm{N}=376$ isolated oocytes $(\mathrm{B}=5$ females), subjected to 8 different concentrations (Figure 6A). Oocytes were sorted based on the amount of phospho-MAPK (Figure 6B). At 1,10-PA $1 \mathrm{mM}, 67 \%$ of oocytes exhibited medium amount of phospho-MAPK (31\% to $60 \%$ ); and at $2 \mathrm{mM}$, high proportion of cells (94\%) exhibited high amount of phospho-MAPK (61\% to $100 \%) .1,10-\mathrm{PA}$ generated partial and gradual response of MAPK since both states of MAPK can be found within the same oocytes. In contrast, when PG-treated oocytes were analysed individually, the two forms of MAPK were never found together in one cell, in a switch-like manner reported by Ferrell et al. ${ }^{25}$

The amount of phospho-MAPK was averaged, fitted and merged (Figure 6C). Low concentrations of 1,10-PA (up to $0.5 \mathrm{mM}$ ) did not generate MAPK response. Within these conditions, no statistical difference was observed with negative control (Figure 6C, area c). 1,10PA $1 \mathrm{mM}$ activated $58.3 \%$ of MAPK $( \pm 2.6 \%$; $\mathrm{P}<0.001^{* * \mathrm{~b}}$ vs other concentrations) and 2 mM led to phosphorylate $78.5 \%$ of MAPK/Erk $\left( \pm 2.9 \% ; \mathrm{P}<0.001^{* * * a}\right.$ vs other concentrations). Using fitting, $\mathrm{Ka}=0.869 \pm 0.063$ and the Hill coefficient was obtained as $n_{H}=6.305 \pm 1.496$ to characterise cell response (MAPK phosphorylation) as a function of variable stimulation (1,10-PA concentration). This value indicated that MAPK response observed in 1,10-PA was ultrasensitive.

In 1,10-PA treated oocytes, MAPK recruitment remains gradual and partial. These observations may reinforce the crucial role of positive feed-back in the building of the All-orNone response, as previously observed 25 (Figure 7A, B, for theoretical purposes, MAP3K-MAP2K-MAPK were respectively noted $\mathrm{x}, \mathrm{y}$ and z).5,25 Nevertheless, here we report that ultrasensitive response of MAPK may be driven in absence of feedback loop, through different regulation motif. Since phosphorylation of MAPK induced by 1,10-PA relies on MAP2K activation but not upon MAP3K, it might be hypothesized that 1,10-PA affects substrate recognition of a zinc sensitive MAP2K-upstream phosphatase, whose inhibi-
A

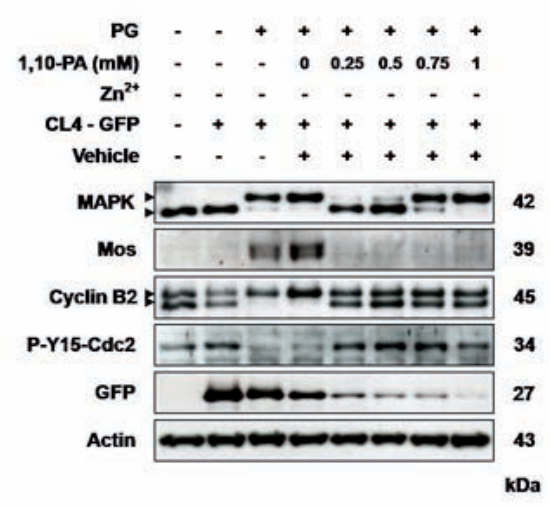

B

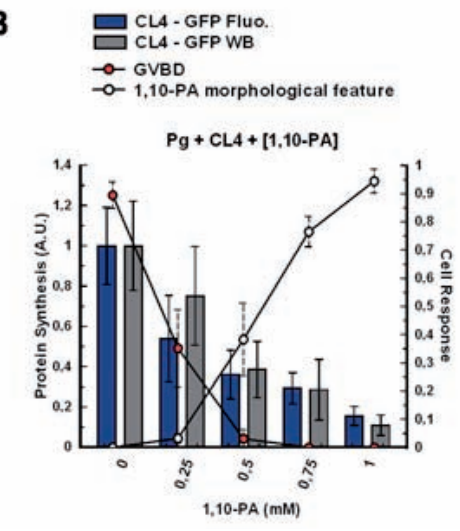

Figure 5. Dose-dependent effect of 1,10 PA on heterologous expression of GFP in Xenopus oocytes. 1,10-PA gradual concentrations were applied on oocytes injected with GFP-CL4 mRNA and stimulated with progesterone (Pg) (A). $0.25 \mathrm{mM} \mathrm{1,10-PA}$ was sufficient to prevent Mos synthesis and both MAPK and MPF pathway activation. GFP-CL4 accumulation was progressively impaired by increasing concentrations $(0.5$ to $1 \mathrm{mM}, \mathrm{B})$. Inhibition of protein synthesis was dose-dependent and correlated with inhibition of PGinduced GVBD.
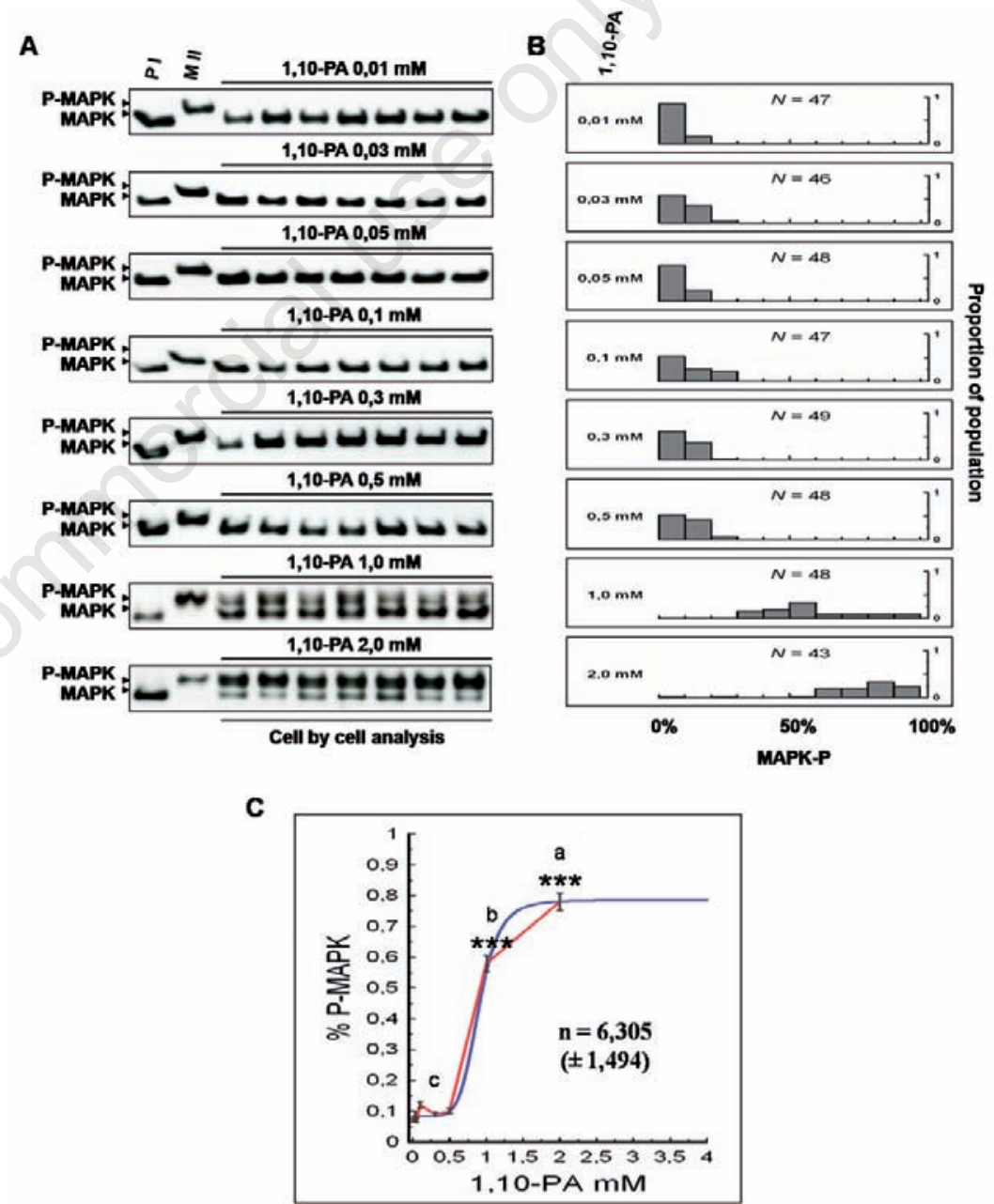

Figure 6. Cell by cell analysis discriminates MAPK/Erk ultrasensitive response in absence of feedback. MAPK phosphorylation profile was analyzed in 376 individual cells, subjected to increasing concentrations of 1,10 PA (A). (B) Proportion of oocytes was sorted depending on the percentage in Phospho-MAPK (MAPK-P). In the graph, $\mathrm{N}$ represents the number of analysed cells for each concentration in 1,10-PA for which P-MAPK percentages were finally averaged and traced (C). No statistical differences were observed between values from area $c$ while highly significant differences were noticed between value a $\left(^{* *}\right)$ vs value $\mathrm{b}\left({ }^{* * *}\right)$ vs values from area c. Experimental curve was fitted (red) and merged (blue) to determine Hill coefficient (nH) linking 1,10-PA and MAPK. 
tion drives the activation of MAP2K, independently of MAP3K. Similar mechanisms, closely related to a feed-forward loop, were described in mice. In this complex, Mos has been proposed to phosphorylate and inactivate a MAP2K-phosphatase. ${ }^{34}$

In order to rationalize the observed value of $\mathrm{n}_{\mathrm{H}}=6.3$ we argue on the basis of our previous theoretical work on cooperativity within the MAP3K-MAP2K-MAPK network. ${ }^{5}$ There we have found that an additional indirect interaction akin to a feed-forward loop involving the down-regulation of either a MAP2K-phosphatase (as shown schematically in Figure 7C) or, alternatively of a MAPK-phosphatase can lead to an increase of cooperativity. Briefly, this effect can be obtained mathematically as follows from the kinetic equations, assuming Michaelis-Menten kinetics:

$$
\begin{aligned}
& \dot{y}_{1}=\frac{V_{6} y_{2}}{K_{6}+y_{2}}-\frac{V_{3} x_{a s} y_{1}}{K_{3}+y_{1}} \\
& \dot{y}_{2}=-\left(\dot{y}_{1}+\dot{y}_{3}\right) \\
& \dot{y}_{3}=\frac{V_{4} x_{a s} y_{2}}{K_{4}+y_{2}}-\frac{V_{5} y_{3}}{K_{5}+y_{3}} \\
& \dot{z}_{1}=\frac{V_{10} z_{2}}{K_{10}+z_{2}}-\frac{V_{7} y_{3} z_{1}}{K_{7}+z_{1}} \\
& \dot{z}_{2}=-\left(\dot{z}_{1}+\dot{z}_{3}\right) \\
& \dot{z}_{3}=\frac{V_{8} y_{3} z_{2}}{K_{8}+z_{2}}-\frac{V_{9} z_{3}}{K_{9}+z_{3}}
\end{aligned}
$$

where the $\mathrm{y}_{\mathrm{i}}$ denote MEK and $\mathrm{z}_{\mathrm{i}}$ MAPK concentrations and only the active and stable form of Mos, xas is considered. The $\mathrm{V}_{\mathrm{i}}$ and $\mathrm{K}_{\mathrm{i}}$ are reaction speeds and equilibrium constants, respectively. The numbering scheme lists the reactions sequentially layer by layer, first all phosphorylations and then all dephosphorylations. Eqs.(2) and (5) are a consequence of the conservation of the total number of the proteins MEK and MAPK, i.e.,

$$
\sum_{i=1,2,3} y_{i}=y_{T}, \quad \sum_{i=1,2,3} z_{i}=z_{T}
$$

Within this phenomenological model a shift of the phosphorylation equilibria due to the action of $\mathrm{P}$ of Figure 7C leads to an effective concentration dependence of the kinetic parameters V5 and V6 on active Mos. In order to take this into account we replace the reaction constants

$$
\begin{aligned}
& V_{5} \rightarrow V_{5}\left(x_{a s}\right)=\frac{V_{5}^{*}}{K^{*}+x_{a s}} \\
& V_{6} \rightarrow V_{6}\left(x_{a s}\right)=\frac{V_{6}^{*}}{K^{*}+x_{a s}}
\end{aligned}
$$
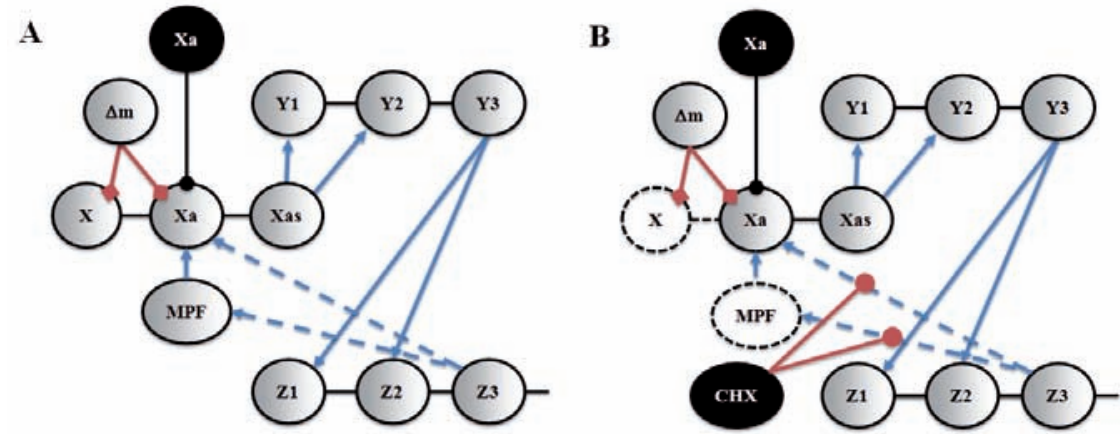

MAPKAll-or-None response, $n_{\mathrm{H}}=35$

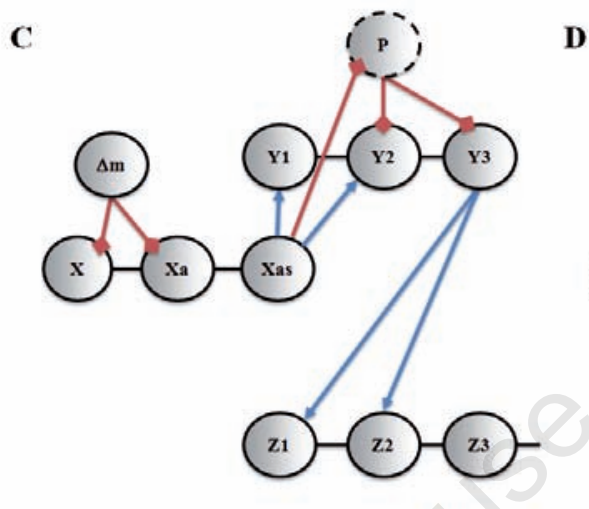

In silico, $\mathbf{n}_{\mathrm{H}}=6$

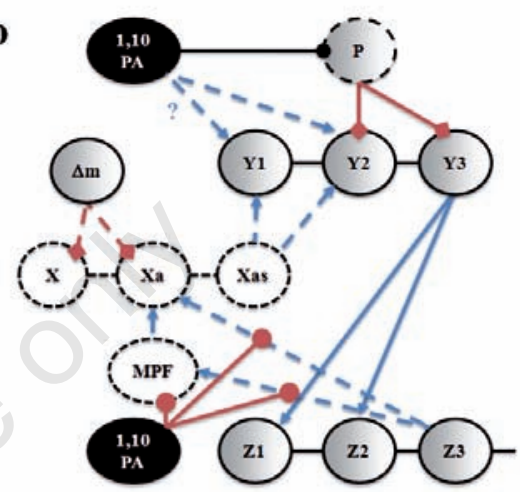

MAPK gradual response, $n_{H}=6.3$

Figure 7. MAP3K-MAPK-MPF network in a simplified diagram model. Nomenclature is as follows: $\mathrm{X}$, unstable inactive Mos; $\mathrm{Xa}$, unstable active Mos; Xas, stable active Mos; Y1,2,3 MAP2K increasing phosphorylation status and likewise Z1,2,3 for MAPK. P stands for MAP2K-phosphatase, whose identity remains undefined and $\Delta \mathrm{m}$ for the degradation machinery. Broken lines represent indirect interaction. Red lines stand for inhibition and blue lines for activation. In absence of hormonal stimulation, injection of Mos/Xa (black sphere) induces typical MAPK/Y3 All-or-None response exhibiting a Hill coefficient $n=35(A)$, while this response is dramatically less sensitive $\left(n_{H}=3\right)$ when $\mathrm{Mos} / \mathrm{Xa}$ is injected in presence of $\mathrm{CHX}(\mathrm{B})$, which breaks direct and indirect feedback from MAPK/Y3 to Mos/Xa (dashed lines). In silico (C), inhibition of $\mathrm{P}$ by Mos is predicted to increase $\mathrm{nH}$ up to 6.(5) Here we propose a model (D) where 1,10 PA i) breaks all routes for feed-back by preventing both accumulation of Mos/Xa and MPF activation but ii) promotes ultrasensitive MAPK/Y3 response $\left(n_{H}=6.35\right)$ through $P$ inhibition.

which increases the cooperativity of the network, as characterized by the Hill coefficient, from four to six. The same effect would be brought about by a similar coupling at the level of MAPK.

\section{Conclusions}

From these a model for 1,10 PA action on the considered network might be proposed where MAP3K/Mos and Raf exert no role (Figure 7D). While abolishing the molecular players involved in feedback loops, 1,10 PA promotes MAPK phosphorylation and activation. Though mammalian MAPK, when expressed in yeasts, become spontaneously phosphorylated, 35 one cannot discard the hypothesis that 1,10 PA may promote conditions for MAP2K autoactivation. Nevertheless, no efficient inherent autophosphorylation capability has been reported or assessed for this protein in vertebrates like amphibian. We furthermore hypothesized that $1,10 \mathrm{PA}$ acts as down-regulator of a MAP2Kphosphatase, since phosphatases offer to the MAPK network flexibility and ability to modulate its responses. ${ }^{36}$ This hypothesis was also based upon the observations of Verlhac and colleagues, ${ }^{34}$ who reported that MAP3K Mos was able to downregulate a MAP2K phosphatase, thus promoting MAP2K activation through two different pathways, being parented to feed forward loop. Such hypothesis was also reinforced by the use in our hands of bpV(Phen), a protein tyrosine-phosphatase inhibitor derived from 1,10 PA, ${ }^{37-39}$ which was able to promote MAPK phosphorylation and 
activation in absence of Mos (Beaujois and Bodart, unpublished observations).

Then, upstream mechanisms of MAPK cascade activation in Xenopus oocytes may hide a coherent feed-forward loop motif, which includes MAP2K downregulation and contributes to the steepness and ultrasensitivity, building strength of stability of the MAPK response.

\section{References}

1. Amiel A, Leclere L, Robert L, et al. Conserved functions for Mos in eumetazoan oocyte maturation revealed by studies in a cnidarian. Curr Biol 2009;19:30511.

2. Dupre A, Suziedelis K, Valuckaite R, et al. Xenopus H-RasV12 promotes entry into meiotic $\mathrm{M}$ phase and cdc2 activation independently of Mos and p42(MAPK). Oncogene 2002;21:6425-33.

3. Baert F, Bodart JF, Bocquet-Muchembled B, et al. Xp42(Mpk1) activation is not required for germinal vesicle breakdown but for Raf complete phosphorylation in insulin-stimulated Xenopus oocytes. J Biol Chem 2003;278:49714-20.

4. Huang CC, Basavappa S, Ellory JC. Volume-activated taurine permeability in cells of the human erythroleukemic cell line K562. J Cell Physiol 1996;167:354-8.

5. Russo C, Beaujois R, Bodart JF, Blossey R. Kicked by Mos and tuned by MPF-the initiation of the MAPK cascade in Xenopus oocytes. HFSP J 2009;3:428-40.

6. Goldbeter A, Koshland DE, Jr. Sensitivity amplification in biochemical systems. Q Rev Biophys 1982;15:555-91.

7. Howard EL, Charlesworth A, Welk J, MacNicol AM. The mitogen-activated protein kinase signaling pathway stimulates mos mRNA cytoplasmic polyadenylation during Xenopus oocyte maturation. Mol Cell Biol 1999;19:1990-9.

8. Keady BT, Kuo P, Martinez SE, et al. MAPK interacts with XGef and is required for CPEB activation during meiosis in Xenopus oocytes. J Cell Sci 2007;120:1093103.

9. Sheng J, Kumagai A, Dunphy WG, Varshavsky A. Dissection of c-MOS degron. Embo J 2002;21:6061-71.

10. Matten WT, Copeland TD, Ahn NG, et al. Positive feedback between MAP kinase and Mos during Xenopus oocyte maturation. Dev Biol 1996;179:485-92.

11. Castro A, Peter M, Magnaghi-Jaulin L, et al. Cyclin B/cdc2 induces c-Mos stability by direct phosphorylation in Xenopus oocytes. Mol Biol Cell 2001;12:2660-71.

12. Csikasz-Nagy A, Kapuy 0, Toth A, et al. Cell cycle regulation by feed-forward loops coupling transcription and phosphorylation. Mol Syst Biol 2009;5:236.

13. Alon U. Simplicity in biology. Nature 2007;446:497.

14. Mangan S, Alon U. Structure and function of the feed-forward loop network motif. Proc Natl Acad Sci U S A 2003;100:11980-5.

15. Cohen EE, Zhu H, Lingen MW, et al. A feedforward loop involving protein kinase Calpha and microRNAs regulates tumor cell cycle. Cancer Res 2009;69:65-74.

16. Watanabe $\mathrm{K}$, Tokumoto $\mathrm{T}$, Ishikawa K. 1,10-Phenanthroline phosphorylates (activates) MAP kinase in Xenopus oocytes. Cell Signal 2003;15:1139-47.

17. Larson DR, Gosse JA, Holowka DA, et al. Temporally resolved interactions between antigen-stimulated IgE receptors and Lyn kinase on living cells. J Cell Biol 2005;171: 527-36.

18. Zhong H, Wu X, Huang H, et al. Vertebrate MAX-1 is required for vascular patterning in zebrafish. Proc Natl Acad Sci USA 2006;103:16800-5.

19. Cailliau K, Le Marcis V, Bereziat V, et al. Inhibition of FGF receptor signalling in Xenopus oocytes: differential effect of Grb7, Grb10 and Grb14. FEBS Lett 2003;5 48:43-8.

20. Riou JF, Clavilier L, Boucaut JC. Early regionalized expression of a novel Xenopus fibroblast growth factor receptor in neuroepithelium. Biochem Biophys Res Commun 1996;218:198-204.

21. Shi DL, Feige JJ, Riou JF, et al. Differential expression and regulation of two distinct fibroblast growth factor receptors during early development of the urodele amphibian Pleurodeles waltl. Development 1992;116:261-73.

22. Flament S, Browaeys E, Rodeau JL, et al. Xenopus oocyte maturation: cytoplasm alkalization is involved in germinal vesicle migration. Int J Dev Biol 1996;40:471-6.

23. Azzi L, Meijer L, Ostvold AC, et al. Purification of a $15-\mathrm{kDa}$ cdk4- and cdk5binding protein. J Biol Chem 1994;269: 13279-88.

24. Chesnel F, Bonnec G, Tardivel A, Boujard D. Comparative effects of insulin on the activation of the Raf/Mos-dependent MAP kinase cascade in vitellogenic versus postvitellogenic Xenopus oocytes. Dev Biol 1997;188:122-33.

25. Ferrell JE, Jr., Machleder EM. The biochemical basis of an all-or-none cell fate switch in Xenopus oocytes. Science 1998;280:895-8.

26. Landrieu I, Hassan S, Sauty M, et al.
Characterization of the Arabidopsis thaliana Arath;CDC25 dual-specificity tyrosine phosphatase. Biochem Biophys Res Commun 2004;322:734-9.

27. Sun L, Chai Y, Hannigan R, et al. Zinc regulates the ability of Cdc25C to activate MPF/cdk1. J Cell Physiol 2007;213:98-104.

28. Wallace RA, Misulovin Z. The role of zinc and follicle cells in insulin-initiated meiotic maturation of Xenopus laevis oocytes. Science 1980;210:928-30.

29. Nikiforova GP, Skoblina MN. [Oocyte maturation and activation in the common frog and the clawed toad under the action of divalent cations]. Ontogenez 1992;23:644-9.

30. Wasserman WJ, Masui Y. Initiation of meiotic maturation in Xenopus laevis oocytes by the combination of divalent cations and ionophore A23187. J Exp Zool 1975;193: 369-75.

31. Hake LE, Mendez R, Richter JD. Specificity of RNA binding by CPEB: requirement for RNA recognition motifs and a novel zinc finger. Mol Cell Biol 1998;18:685-93.

32. Cheng S, Sultana S, Goss DJ, Gallie DR. Translation initiation factor $4 \mathrm{~B}$ homodimerization, RNA binding, and interaction with Poly(A)-binding protein are enhanced by zinc. J Biol Chem 2008;283:36140-53.

33. Volpon L, Osborne MJ, Capul AA, et al. Structural characterization of the Z RINGeIF4E complex reveals a distinct mode of control for eIF4E. Proc Natl Acad Sci USA 2010 23;107:5441-6.

34. Verlhac MH, Lefebvre C, Kubiak JZ, et al. Mos activates MAP kinase in mouse oocytes through two opposite pathways. Embo J 2000;19:6065-74.

35. Levin-Salomon V, Maayan I, AvrahamiMoyal L, et al. When expressed in yeast, mammalian mitogen-activated protein kinases lose proper regulation and become spontaneously phosphorylated. Biochem J 2009;417:331-40.

36. Bhalla US, Ram PT, lyengar R. MAP kinase phosphatase as a locus of flexibility in a mitogen-activated protein kinase signaling network. Science 2002;297:1018-23.

37. Rumora L, Barisic K, Maysinger D, Zanic Grubisic T. BpV (phen) induces apoptosis of RINm5F cells by modulation of MAPKs and MKP-1. Biochem Biophys Res Commun 2003;300:877-83.

38. Rumora L, Hadzija M, Maysinger D, ZanicGrubisic T. Positive regulation of ERK activation and MKP-1 expression by peroxovanadium complex bpV (phen). Cell Biol Toxicol 2004;20:293-301.

39. Cerovac Z, Ban J, Morinville A, et al. Activation of MAPK by potassium bisperoxo(1,10-phenanthroline) oxovanadate (V). Neurochem Int 1999;34:337-44. 